\title{
FUZZY LOGIC BASED INTENTION RECOGNITION IN STS PROCESSES
}

\author{
Hang Lu, Dayou Li, Renxi Qiu \\ Robotics and Smart Systems Research Centre, Institute for Research in Applied Computing, University \\ of Bedfordshire, Park Square, Luton, LU1 3JU
}

\begin{abstract}
This paper represents a fuzzy logic based classifier that is able to recognise human users' intention of standing up from their behaviours in terms of the force they apply to the ground. The research reported focused on the selection of meaningful input data to the classifier and on the determination of fuzzy sets that best represent the intention information hidden in the force data. The classifier is a component of a robot chair which provide the users with assistance to stand up based on the recognised intention by the classifier.
\end{abstract}

\section{Introduction}

A large number of older people suffer from difficulties in sit-to-stand (STS) processes due to the decline of their physical and cognitive capabilities because of aging. There are motorised chairs in the market that can help the older people to complete STS process by rising and reclining. These products, however, can lead the development of the habit of fully relying on the chairs in STS, speeding up the decline of the capabilities. The paper reports a fuzzy logic (FL \} based intention recognition classifier as a part of the efforts to develop a robot chair to allow the provision of assistance to the chair users in STS only when it becomes a desperate need. The system is able to recognise the intention of standing up from the users' behaviours which is represented with the forces they apply to the ground.

The robot chair basically consists of a seat that is able to rise and to recline to assist the user to stand up and a footmat containing a pressure sensor array to the force data. The changes of the force may be caused by the users' movements as the efforts of standing up from the chair. They can also the results of random posture changes of the users. For the former, the assistance would be necessary, but for the later, the provision of the assistance can lead to the situations here the users are pushed over. Therefore, there is a need for recognise the user's intention.

Due to uncertainties existing in the force data in both cases, an FL based point wise classifier was developed to recognise the intention of standing up from the data in the research reported in this paper. The FL-based classifier employs the first-type Sugeno fuzzy inference that performs fuzzy reasoning based on the weighted sum of fuzzified data of each sampling points. The emphasis was on the selection of meaningful input data to the classifier and on the determination of fuzzy sets that can best represent the intention information of the force data.

\section{Background}

The entire STS process can be separated into 6 events according to vertical ground reaction force (GRF), the force humans applied to the ground when standing up from chairs. The 6 events, namely initiation, counter, seat-off, peak, rebound and standing, which are shown in Figure 1, are defined as the following ${ }^{[1]}$ :

1. Initiation $(\mathrm{GRF} \approx 20 \%$ body weight $(\mathrm{BW})$ ) refers to the period from being informed to initiate the STS, to the first deviation of GRF greater than $10 \mathrm{~N}$ captured. This event commonly represents the duration humans take to get mentally prepared for oncoming STS from the neuro-musculo-skeletal perspective.

2. Counter $(\mathrm{GRF} \approx 12 \% \mathrm{BW})$ is mainly caused by early lifting of thighs from seat by contracting the hip flexor muscles while upper torso mostly remains in its original position.

3. Seat-off (GRF $\approx 60 \%-70 \% \quad \mathrm{BW})$ is signified by the moment when humans' buttocks separate from seat. The captured GRF increases dramatically in this event which is reported to appear after $1.0 \mathrm{~s}$ from the beginning of STS.

4. Peak $(\mathrm{GRF} \approx 116 \% \mathrm{BW})$ is simply denoted as the event where the captured GRF reaches its maximum.

5. Rebound (GRF $\approx 80 \% \mathrm{BW}$ ) represented by a less-than-body-weight GRF observed after the peak event which can also be understood as the tendency of both feet leaving the ground.

6. Standing $(\mathrm{GRF}=100 \% \mathrm{BW})$ refers to the period from rebound to steady standing up.

Etnyre and Thomas ${ }^{[1]}$ conducted tests on 100 individuals to measure GRF under four possible postures, namely, arms-free, arms-crossed, hands-on-knees and hands-on-armrest. It was found that the average normalised GRFs were similar for the first 3 postures in the 6 events. In contrary, with hands-on-armrest posture, significantly less normalised GRFs were found, 
especially in the event of seat-off. This is mainly because the pushing off force exerted onto arm-rest will contribute to vertical lifting motions hence reducing the GRF, which is further confirmed by related work conducted ${ }^{[2]}$. Most of functional limitations and deficits were spotted under hands-free condition, where humans have to primarily rely on lower limbs to provide the strength and stability to complete the STS transition.

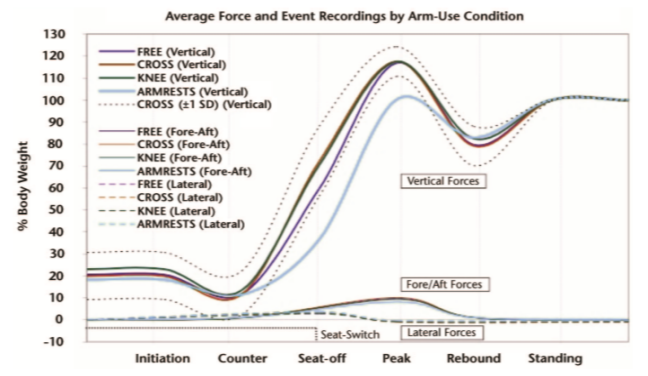

Figure 1. GRF to the percentage of body weight in STS and

$$
\text { the } 6 \text { events }
$$

The maximum torques at knees, buttocks and ankles are generated at the instant when buttocks separated from seat (seat-off), with the only physical contact being the feet against the ground (hands-free conditions) ${ }^{[3]}$. It is critical for the robot chair to recognise humans' intention of standing up before the seat-off event. The delayed recognition could lead to situations where assistance is unavailable at the point it is needed.

Human intention recognition in STS processes needs to take the followings into account:

1. Safety is primarily critical to the intention recognition. In cases where human users do not intend to stand up, if the robot chair mis-interprets subjects' behaviours and actuates the lifting mechanism, it could drive the elderly users into panic situations and potentially cause injuries.

2. Accuracy is another issue. The robot chair aims at supporting subjects' STS movements at the point where he/she is not able to perform unaided STS. Although assistance provided at the time when not needed can also help the subject to stand up, it will discourage him/her from using own motor functions, leading to lose of function eventually.

3. Robustness is the third concern. Human users, even the same user, will not follow the same pattern when performing STS movements. A certain amount of uncertainties need to be dealt with.

4. Timing also plays a critical role. Intention recognition has to be completed within a limited time scale. STS process commonly takes from $1.91 \mathrm{~s}$ to $2.30 \mathrm{~s}$ for completely able-bodied healthy adults to perform in natural speed depending on several related studies $^{[1]}{ }^{[4-5]}$. Although STS duration is proved to be longer for able-bodied elderly users, some will not resist more than $3.00 \mathrm{~s}$ until he/she sits back to seated position. In both cases, the first about $1.00 \mathrm{~s}$ is the period when STS is initiated and hence the intention recognition must be done within this period to support the followed-up motion of stand up. Otherwise incapable subjects will not reach the point of seat-off without any external assistance.

\section{Centre of Pressure}

Human users' intentions can be sorted as the following three:

1. Intend to rise up from seated position and capable of proceeding the entire STS movement, denoted as Successful STS movement.

2. Intend to rise up despite physiological condition does not allow to do so, denoted as Unsuccessful STS movement.

3. Not intend to rise up, denoted as Unintended movement.

Being the key to successfully meet the four requirements, intention recognition must be done based on feasible and reliable features that distinguishing different intentions. In the related work on biomechanics of STS, because the centre of body mass is posterior, the centre of pressure (COP) transiently shifts backward after the initiation of STS movement. Afterwards, the COP shifts forward for the sake of the dominating upward movement. Before the completion of STS movement, the COP again shifts slightly backward. Meanwhile, ankles are consistently generating longitudinal and lateral motions, named ankle strategies, to adjust and maintain the balance along with the entire STS process. At last, a stable standing position is achieved following a postural adjustment (Hughes et al, 1996).

The study on shift of Centre of Pressure (COP) is conducted from the three aspects, a) Magnitude of change in COP, b) Longitudinal shift of COP, c) Lateral shift of COP. Because all the sensory data is collected from footmat, any sort of COP captured here does not directly imply Centre of Gravity (COG) of the subject. Figures 2 to 4 show the 3 parameters, respectively, with the data obtained through experiments of this study. Magnitude of change in COP shows muscle activation using Electromyogram (EMG) in the sense of determining the intensity of activity over a period of time ${ }^{[6]}$. However, it can be seen from Figure 2 that Unintended random movements are not significantly different from the rest two in the early stage. 
As related work $^{[4]}$ suggested, a smooth transition of COG in Longitudinal directions is supposed to be observed from the Successful STS movement. Longitudinal shift of COG is minimal as the force applied to the footmat is primarily vertical GRF to generate upwards motion, with the follow-up leaning forward motion given by upper torso. After this, with the upper torso straightening after leaning forward in first 4 STS events. Again, neither forward motion nor COG of subject is able to be manifested by trend of Longitudinal shift appearing on the footmat.

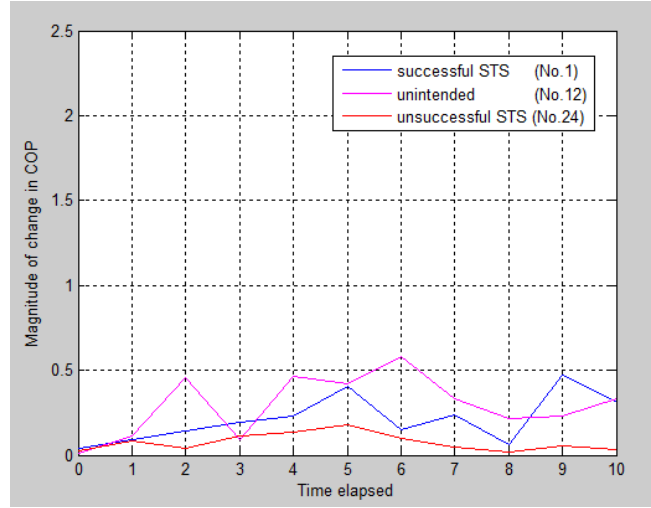

Figure 2. Magnitude of change in COP in three movements

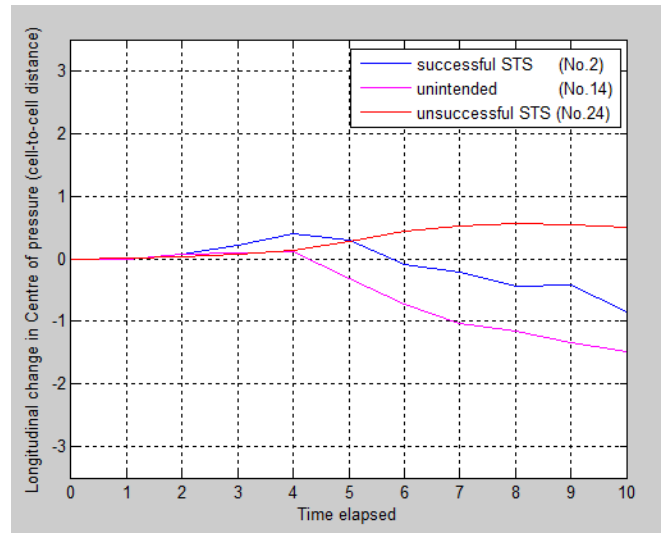

Figure 3. Longitudinal shift of COP in three movements

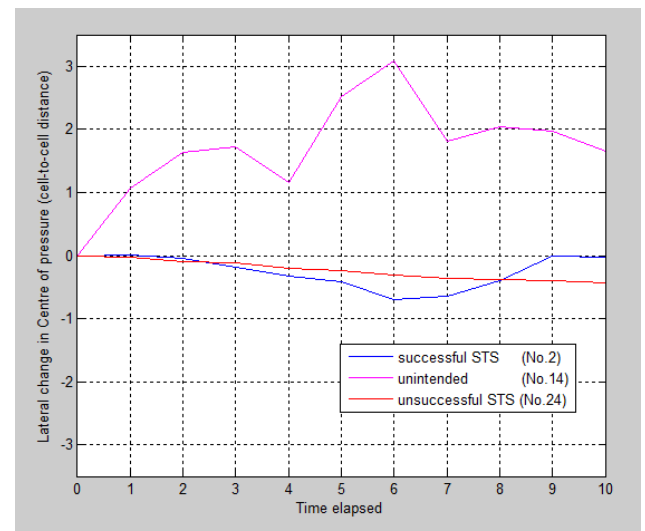

Figure 4. Plot of Lateral shift of COP in three movements
Figure 4 clearly demonstrates that Lateral shift of COP captured in Unintended movements shows significantly greater absolute values when compared with the other two. To further confirm this, the lateral shift of COP from all recorded data on the three types of movements are plotted respectively as Figures 5 to 7 . With the vertical axis fixed at same range, it is obvious that subject's feet shift laterally far more frequently when performing Unintended movements.

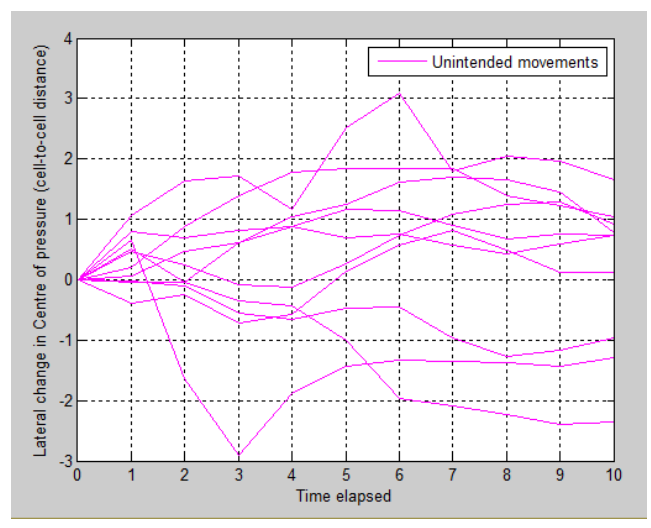

Figure 5. Lateral shift of COP in all Unintended movements

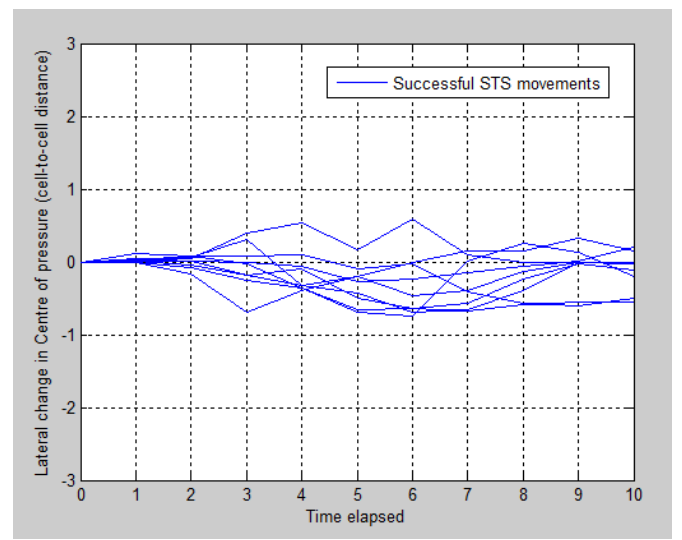

Figure 6. Lateral shift of COP in all Successful movements

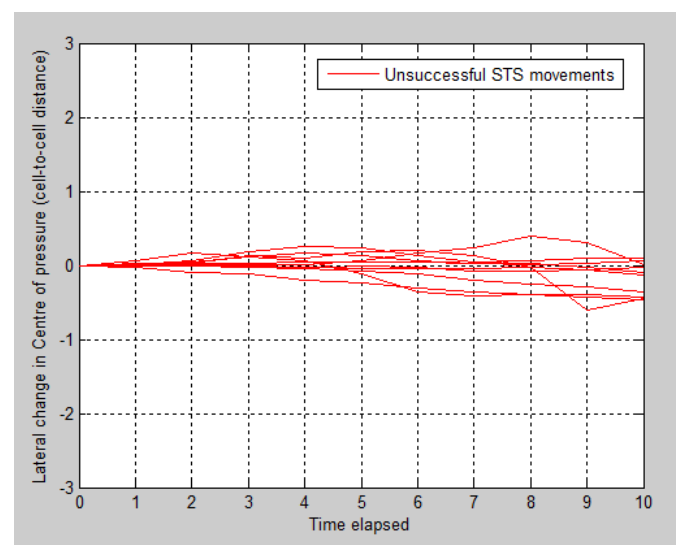

Figure 7. Lateral shift of COP in all Unsuccessful movements 
It could be concluded change of Lateral shift of COP in Unintended motions is distinctively higher, comparing to the Successful STS and Unsuccessful STS movements.

\section{Fuzzy Logic Based Point-wise Classifier}

Fuzzy logic is employed to implement the classification aiming to recognise human intention via differentiating Intended and Unintended movements, due to the following reasons:

- Lack of access to exact modelling of human biomechanical model as well as mechanical geometries

- Uncertainties existing in human behavioural data of STS movements. The Lateral COP distributions displacement, will not be exactly the same among different users considering vast difference in health conditions. Even for the same user, health condition can vary from time to time and will not keep the same positioning and posture prior to standing up. All of these contribute to the uncertainties of Lateral shift of COP. Certain uncertainties have been observed through experiments carried out in this research.

The point-wise classification approach makes decisions in the sense of fuzzification at each sampling point. The distribution of actual data of LS of COP is shown in Figure 8, with separating the 2 types of movements, Unintended and Intended STS.

Among 200 Intended movement samples, there are merely a minority of points (22 out of 200) having an absolute LS of COP larger than 0.5 units, which not only helps to define fuzzy sets of the FL based intention recognition classifier, but also contributes to determine the truth degrees of the fuzzy rules of the system. The fuzzy sets of A, B and C for LS of COP were defined as shown in Figure 9.

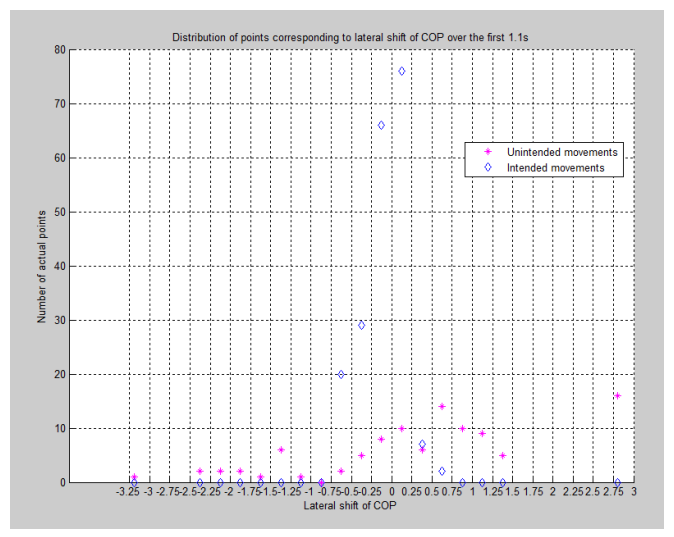

Figure 8. Distribution of LS of COP in Unintended (100 points) and Intended (200 points) movements over the early stages

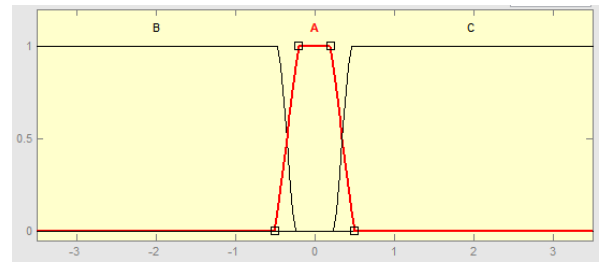

Figure 9. Fuzzy sets of A, B and C regarding to LS

Fuzzy rules were defined in an intuitive way. For a change in lateral direction that is outside of $[-1,1]$, it is more likely the movement to be classified as "Unintended". Therefore, the following fuzzy rules were obtained for classifying intentions into "Intended" and "Unintended" categories:

Rule 1: If $\mathrm{LS}=\mathrm{A}$, then intention $=$ "Intended", with a truth degree of $0.89(178 / 200)$.

Rule 2: If $\mathrm{LS}=\mathrm{B}$, then intention $=$ "Unintended", with a truth degree of 0.76 (37/49).

Rule 3: If $\mathrm{LS}=\mathrm{C}$, then intention $=$ "Unintended", with a truth degree of 0.67 $(34 / 51)$

Rule 4: If $\mathrm{LS}=\mathrm{A}$, then intention $=$ "Unintended", with a truth degree of 0.11 (22/ 200).

Rule 5: If $\mathrm{LS}=\mathrm{B}$, then intention = "Intended", with a truth degree of $0.24(12 / 49)$.

Rule 6: If $\mathrm{LS}=\mathrm{C}$ then intention = "Intended", with a truth degree of $0.67(34 / 51)$.

The truth degrees of Rules 1, 5 and 6 were determined based on the number of Intended samples that were in the range of the fuzzy sets A, B and C, respectively. Those of Rules 2 to 4 were obtained with the similar manner but considering unintended samples.

The final output of the classification is the weighted average of outputs of all fired rules, according to the zero-order Sugeno fuzzy inference, which can be represented with the following equation:

$Z_{f}=\frac{\sum_{i=1}^{N} w_{i} Z_{i}}{\sum_{i=1}^{N} w_{i}}$

\section{Example:}

The following shows how point-wise classification works based on the fuzzy sets and rules. Given the value of LS as 0.43 , which is the sampled value at a point of 0.9 seconds of an Unintended movement. Fuzzy rules of 1, 3, 4 and 6 were fired, as shown in Figure 10.

With Equation (1), the output of each of fired rule can be calculated as:

Rule 1 (Intended), $\frac{0.5-0.43}{0.5-8.25} \times W_{m f p A}=$ $0.28 \times 0.89=0.255$, where $\frac{0.550 .43}{0.5-0.25}$ is the fuzzification of sample value on fuzzy set A.

Rule 4 (Unintended), $\frac{0.43-0.25}{0.5-0.25} \times W_{m f p A^{\prime}}=$ $0.72 \times 0.11=0.079$,

Rule 3 (Unintended), $\frac{0.43-0.25}{0.5-0.25} \times W_{m f p C^{\prime}}=$ $0.72 \times 0.67=0.482$, 
Rule $6 \quad$ (Intended), $\quad \frac{0.5-0.43}{0.5-0.25} \times W_{m f p c}=$ $0.28 \times 0.33=0.092$,

where $W_{m f p A}, W_{m f p A^{\prime}}, W_{m f p c^{\prime}}$ and $W_{m f p c}$ are corresponding truth degrees of the fired rules.

The weighted sum of corresponding rules: Probability of classifying the sample point as "Unintended" is $0.48+0.079=0.569$

Probability of classifying the sample point as "Intended" is $0.092+0.23=0.322$

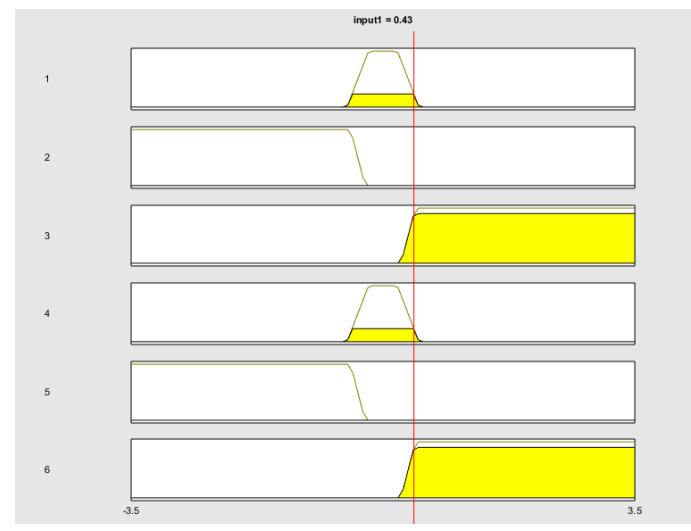

Figure 10. Rules fired by Example 4.1

Table 1: Complete point-wise classification of all sample points

\begin{tabular}{|r|r|r|}
\hline Sample points No. & Unintended & Intended \\
\hline 1 & 0.11 & 0.89 \\
\hline 2 & 0.11 & 0.89 \\
\hline 3 & 0.11 & 0.89 \\
\hline 4 & 0.11 & 0.89 \\
\hline 5 & 0.11 & 0.89 \\
\hline 6 & 0.3622 & 0.3528 \\
\hline 7 & 0.4918 & 0.318 \\
\hline 8 & 0.4456 & 0.3314 \\
\hline 9 & 0.569 & 0.323 \\
\hline 10 & 0.6523 & 0.2493 \\
\hline Classification outputs & 3.0709 & 6.0245 \\
\hline
\end{tabular}

\section{Evaluation and discussions}

Criteria for comparing the three approaches of classification are set at the beginning of this chapter. The followings are defined as the measures of the criteria:

- False matching rate of unintended movements to intended ones (FMR) is measure for the safety. The rate should be minimised as the false matching will cause the assist actions of the robot chair to take place, which may force the user who has no intention to stand up and lead to a fall.

- True matching rate of intended to intended (TMR) is the measure for the accuracy. This should be maximised as the aim of the classification is to find out the user's need for assistance in STS process.

- Variety in sample data (VD) in conjunction with FMR and TMR are used to measure the robustness. High variety means high uncertainty level in sample data. Together with FMR and TMR, they reflect the capabilities of handling the uncertainty.

- $\quad$ Responding time of the classification (RT) reflects the time criterion. The classification must be done at the end of first 1.1 seconds period.

Tests with 2-fold testing data and with pure testing data were undertaken in the way that a set of 10 Intended movements data was fed to the FL-based classifier, followed by a set of 10 Unintended data, and then another set of 10 Intended data. The results with 2 -fold data are shown in Table 2. In each section of the table, the first row are the results of Equation (1) regarding Unintended and the second row as Intended. All the Intended movements were correctly classified as Intended, but No. 14, 15 and 17 that are Unintended movements data were classified as Intended. Tests were also performed with pure test data. The results are recorded in Table 3. Of all Intended movements, $30 \%$ of Intended movements were incorrectly classified as Unintended. As to the Unintended movements, $20 \%$ were classified as Intended.

As introduced early, FMR is primarily highlighted for safety concerns of the robot chair. It will cause safety issues in the scenarios where subject is intended to simply change his/her posture but the actuator mechanism arises the entire seat due to the false matching of intention. The top-right corner of each confusion matrix above shows the FMRs of the different approaches. With 2-fold test data FMR were recorded as $30 \%$. With pure test data, the FMRs became $20 \%$.

TMR is used as another critical measure to benchmark the accuracy. Referring to the bottom-right corner of the above confusion matrices, the TMR with 2-fold input test data were recorded as $100 \%$, whilst with pure test data, the FMRs became $70 \%$.

VD is another measure of performance of the classification approaches. VD is considered high in the datasets as movements were executed naturally to introduce realistic uncertainties.

The RT is considered through 2 aspects, the captured time span of input data and processing time of fuzzy reasoning. The time duration of input data is limited to 1.1 seconds. In addition to this, the average processing times of fuzzy reasoning were recorded as 0.077 seconds which is rather negligible comparing to the 1.1 seconds of input data capturing. 
Table 2: Results of point-wise classification 2-fold data

\begin{tabular}{|r|r|r|r|r|r|r|r|r|r|r|r|}
\hline & 1 & 2 & 3 & 4 & 5 & 6 & 7 & 8 & 9 & 10 \\
\hline 1 & 0.2484 & 0.3141 & 0.2832 & 0.3327 & 0.4918 & 0.0884 & 0.3198 & 0.3141 & 0.2422 & 0.1186 \\
\hline 2 & 0.5622 & 0.5036 & 0.5241 & 0.5348 & 0.4475 & 0.7107 & 0.5094 & 0.5036 & 0.6460 & 0.5890 \\
\hline
\end{tabular}

\begin{tabular}{|c|c|c|c|c|c|c|c|c|c|c|}
\hline & 11 & 12 & 13 & 14 & 15 & 16 & 17 & 18 & 19 & 20 \\
\hline 1 & 0.6032 & 0.6132 & 0.7510 & 0.4248 & 0.4209 & 0.5607 & 0.1654 & 0.6521 & 0.6700 & 0.6093 \\
\hline 2 & 0.3703 & 0.3794 & 0.2490 & 0.4253 & 0.4019 & 0.3539 & 0.5269 & 0.3212 & 0.3300 & 0.3483 \\
\hline
\end{tabular}

\begin{tabular}{|r|c|c|c|c|c|c|r|r|r|r|r|r|r|}
\hline & 21 & 22 & 23 & 24 & 25 & 26 & 27 & 28 & 29 & 30 \\
\hline 1 & 0.1322 & 0.0834 & 0.2860 & 0.1012 & 0.0960 & 0.1018 & 0.1014 & 0.1182 & 0.2295 & 0.2054 \\
\hline 2 & 0.6705 & 0.6746 & 0.4534 & 0.8189 & 0.7766 & 0.8237 & 0.8205 & 0.6464 & 0.4422 & 0.6101 \\
\hline
\end{tabular}

Table 3: Results of point-wise classification with pure test data

\begin{tabular}{|c|c|c|c|c|c|c|c|c|c|c|}
\hline & 1 & 2 & 3 & 4 & 5 & 6 & 7 & 8 & 9 & 10 \\
\hline 1 & 0.5933 & 0.6347 & 0.5654 & 0.2044 & 0.6700 & 0.6999 & 0.4355 & 0.5380 & 0.4754 & 0.3652 \\
\hline 2 & 0.3346 & 0.3366 & 0.3466 & 0.7242 & 0.3300 & 0.2575 & 0.5035 & 0.4620 & 0.4930 & 0.5619 \\
\hline
\end{tabular}

\begin{tabular}{|r|c|c|c|c|c|c|r|r|r|r|r|r|r|r|}
\hline & 11 & 12 & 13 & 14 & 15 & 16 & 17 & 18 & 19 & \multicolumn{1}{c|}{17} \\
\hline 1 & 0.5945 & 0.5237 & 0.5808 & 0.6087 & 0.4685 & 0.6700 & 0.6700 & 0.7600 & 0.4076 & 0.6700 \\
\hline 2 & 0.3630 & 0.3286 & 0.3241 & 0.3429 & 0.3897 & 0.3300 & 0.3300 & 0.2400 & 0.4045 & 0.3300 \\
\hline
\end{tabular}

\begin{tabular}{|r|c|c|c|c|c|c|r|r|r|r|r|r|}
\hline & 21 & 22 & 23 & 24 & 25 & 26 & 27 & 28 & 29 & 30 \\
\hline 1 & 0.0926 & 0.4748 & 0.2806 & 0.1474 & 0.3750 & 0.4370 & 0.0974 & 0.0974 & 0.3776 & 0.3615 \\
\hline 2 & 0.7493 & 0.3221 & 0.4865 & 0.6019 & 0.4393 & 0.4977 & 0.7881 & 0.7881 & 0.4928 & 0.5616 \\
\hline
\end{tabular}

\section{Conclusion}

A fuzzy logic based point wise classifier was developed to recognise the intention of standing up of human users from a robot chair. The classification results are used by the chair to decide the provision of assistance to the users for helping them to complete the STS processes. The classifier achieved reasonable good results and also leave rooms for the further development.

\section{REFERENCES}

[1] B. Etnyre and D.Q. Thomas, "Event standardization of sit-to-stand movements," Physical Therapy, vol. 87, no. 12, pp. 1651-1666, December 2001

[2] T. Yamada and S. Demura, "Relationships between ground reaction force parameters during a sit-to-stand movement and physical activity and falling risk of the elderly and a comparison of the movement characteristics between the young and the elderly", Archives of Gerontology and Geriatrics, vol. 48, no. 1: 73-77, Feb. 2009

[3] M.A. Hughes, B.S. Myers, and M.L. Schenkman, "The role of strength in rising from a chair in the functionally impaired elderly," Journal of biomechanics, 29, no. 12, December 1996, pp. 1509-1513
[4] K.M. Kerr, J.A. White, D.A. Barr, and R.A.B. Mollan, "Analysis of the sit-stand-sit movement cycle in normal subjects," Clinical Biomechanics, vol. 12, no. 4, pp. 236-245, June 1997

[5] J. Schlicht, D.N. Camaione, and S.V. Owen, "Effect of intense strength training on standing balance, walking speed, and sit-to-stand performance in older adults," The Journals of Gerontology Series A: Biological Sciences and Medical Sciences, vol. 56, no. 5, pp. 281-286, May 2001

[6] J.H. Bae, and I. Moon, "Design of electric assist-standing chair for persons with disability design of electric chair to assist person with disability in stand up and sitting down," 2010 International Conference Control Automation and Systems (ICCAS), pp. 574-575, October 2010

[7] M.S. Tremblay, R.C. Colley, T.J. Saunders, G.N. Healy, and N. Owen, "Physiological and health implications of a sedentary lifestyle," Applied Physiology, Nutrition, and Metabolism, vol. 35, no. 6, 725-740, 2010

[8] M.J. Coggrave, and Rose, L.S, "A specialist seating assessment clinic: changing pressure relief practice," Spinal Cord, no. 41, pp. 692-695, 2003 